\title{
Asiatic acid inhibits lactate-induced cardiomyocyte apoptosis through the regulation of the lactate signaling cascade
}

\author{
CHANGZHENG GAO $^{1,2}$, FEN WANG $^{2}$, ZHIQIANG WANG $^{3}$, JING ZHANG $^{2}$ and XIANGJUN YANG ${ }^{1}$ \\ ${ }^{1}$ Department of Cardiology, The First Affiliated Hospital of Soochow University, Suzhou, \\ Jiangsu 215006; Departments of ${ }^{2}$ Cardiology and ${ }^{3}$ Cardiothoracic Surgery, The Affiliated Hospital of \\ Jiangnan University, Wuxi 4th People's Hospital, Wuxi, Jiangsu 214062, P.R. China
}

Received December 16, 2015; Accepted October 12, 2016

DOI: $10.3892 /$ ijmm.2016.2783

\begin{abstract}
The lactate signaling cascade has recently been linked to mitochondrial energy metabolism and cardiomyocyte apoptosis in several cardiovascular diseases. Asiatic acid (AA) exhibits a variety of pharmacological effects, including antioxidant and anti-apoptotic effects. In this study, we investigated the protective effects of AA against the lactate-induced apoptosis of cardiomyocytes, as well as its mechanisms of action. Neonatal rat cardiomyocytes were pre-treated with $20 \mu \mathrm{M}$ AA for $24 \mathrm{~h}$, followed by exposure to $20 \mathrm{mM}$ lactate for a further $24 \mathrm{~h}$. Cell viability was determined by a cell counting kit-8 (CCK-8) assay. Cell apoptosis, reactive oxygen species (ROS) levels and mitochondrial membrane potential $(\Delta \psi \mathrm{m})$ were evaluated by flow cytometry or fluorescence microscopy. The expression levels of mitochondrial monocarboxylate transporter 1 (MCT1) and cytoplasmic cytochrome $c$, cleaved caspase- 9 and caspase- 3 were assayed by western blot analysis. Our results revealed that AA significantly inhibited lactate-induced apoptosis, intracellular ROS generation and the loss of $\Delta \psi \mathrm{m}$. AA also increased the expression of mitochondrial MCT1 and reduced the expression of cytoplasmic cytochrome $c$, cleaved caspase- 9 and caspase- 3 in the lactate-stimulated cardiomyocytes. To the best of our knowledge, our data demonstrate for the first time that AA plays a cytoprotective role in lactate-induced apoptosis by regulating the lactate signaling cascade, involving the inhibition of oxidative stress and mitochondria-dependent caspase activation, as well as the upregulation of mitochondrial MCT1 expression.
\end{abstract}

\section{Introduction}

Cardiovascular disease is the leading cause of morbidity and mortality in the modern world. It is now evident that cardiomyo-

Correspondence to: Dr Xiangjun Yang, Department of Cardiology, The First Affiliated Hospital of Soochow University, 188 Shizi Street, Suzhou, Jiangsu 215006, P.R. China

E-mail: yang_xiangjun@163.com

Key words: asiatic acid, cardiomyocyte, apoptosis, oxidative stress, mitochondria, monocarboxylate transporter 1 cyte apoptosis plays a key role in the pathogenesis of various cardiovascular diseases, including myocardial infarction (1), ischemia/reperfusion injury (2), dilated cardiomyopathy (3) and end-stage heart failure (4). Apoptosis is a highly regulated program of cell death and can be activated in cardiomyocytes by multiple stressors, including cytokines (5), oxidative stress (6) and DNA damage (7). Previous studies have demonstrated that the inhibition of apoptosis exerts cardioprotective effects and can prevent the development of heart failure $(8,9)$.

Lactate is produced as the result of glycolysis in the cytosol and is balanced by oxidation in the mitochondria. This process of the intracellular lactate shuttle is mediated by monocarboxylate transporter 1 (MCT1) on the mitochondrial membrane and plays an important role in the energy metabolism of cardiomyocytes $(10,11)$. However, it is now known that lactate is produced continuously under fully aerobic conditions. Lactate functions as an important signaling molecule to regulate intracellular reactive oxygen species (ROS) the generation and the lactate signaling cascade (12). Under normal physiological conditions, low levels of ROS upregulate the expression of MCT1 by activating several transcription factors, which, in turn, facilitate the transport of lactate into the mitochondria for oxidative metabolism (13). However, under pathological conditions, the intracellular lactate concentration increases several fold and promotes the excessive generation of ROS. High levels of ROS can cause oxidative stress and mitochondrial damage, which lead to the activation of mitochondrial-dependent apoptosis (14). A significant association has been identified between the lactate signaling cascade and cardiovascular diseases, such as myocardial infarction (15), ischemic/reperfusion injury (16), atrial fibrillation (17) and heart failure (18). Therefore, targeting the regulation of the lactate signaling cascade may be a promising therapeutic approach for the treatment of multiple cardiovascular diseases.

Asiatic acid (AA) is a triterpenoid derived from Centella asiatica. Pharmacologically, it is used as an antioxidant (19) and anti-inflammatory (20) agent, and has been shown to exert neuroprotective (21) and hepatoprotective (22) effects. Recently, Si et al (23) reported that AA attenuated cardiac hypertrophy by blocking transforming growth factor- $\beta 1$ (TGF- $\beta 1$ ) mediated hypertrophic signaling. Zhang et al (24) reported that AA prevented C2-ceramide-induced neuronal injury by inhibiting mitochondrial-dependent apoptosis. Therefore, in this study, we hypothesized that AA may inhibit cardiomyocyte apoptosis. 
To examine this hypothesis, we investigated the anti-apoptotic effects and mechanisms of action of AA using an in vitro model of lactate-induced cardiomyocyte apoptosis.

\section{Materials and methods}

Reagents. The following reagents were obtained from the indicated commercial sources. Purified natural product of AA (97\%), dimethyl sulfoxide (DMSO), sodium lactate and 2',7'-dichlorofluorescein-diacetate (DCFH-DA) were obtained from Sigma-Aldrich (St. Louis, MO, USA). 5,5',6,6'-tetrachloro1,1',3,3'-tetraethyl-imidacarbocyanine iodide (JC-1) was obtained from BD Biosciences (San Jose, CA, USA). The Annexin V-fluorescein isothiocyanate (FITC)/propidium iodide (PI) staining kit and the in situ cell death detection kit were obtained from Roche Applied Science (Mannheim, Germany). Dulbecco's modified Eagle's medium (DMEM) and fetal bovine serum (FBS) were obtained from Gibco-BRL Life Technologies, Inc. (Carlsbad, CA, USA). The cell counting kit-8 (CCK-8) assay kit was obtained from Dojindo Laboratories (Kumamoto, Japan). The anti-cleaved caspase-9 (9509S), anti-cleaved caspase-3 (9661S), anti-cytochrome $c$ oxidase polypeptide IV (COX4; 4844S) and anti- $\beta$-actin (4967S) primary antibodies, and HRP-conjugated secondary antibody (7074S) were obtained from Cell Signaling Technology (Danvers, MA, USA). The anti-cytochrome $c$ primary antibody (sc-13560) was obtained from Santa Cruz Biotechnology, Inc. (Santa Cruz, CA, USA). The anti-MCT1 primary antibody (AB3540P) was obtained from Chemicon International (Temecula, CA, USA). The cytoplasmic and mitochondrial protein extraction kits were obtained from Keygen Biotechnology (Nanjing, China). Unless otherwise indicated, all chemicals and materials were purchased from Sigma-Aldrich.

Cell culture and treatment. Primary cultures of ventricular myocytes were prepared from neonatal Sprague-Dawley rats. All animal care and experimental protocols complied with the Guide for the Care and Use of Laboratory Animals, published by the US National Institutes of Health (NIH Publication no. 85-23, revised 1996) and were approved by the Animal Ethics Committee of First Hospital Affiliated to Soochow University. All efforts were made to minimize animal suffering and the number of animals used. As previously described (23), 20 neonatal Sprague-Dawley rats (1-2 days of age, weighing, 5-8 g; from the Experimental Animal Center of Soochow University, Suzhou, China) were anesthetized by isoflurane. The hearts were then immediately removed under aseptic conditions and washed in $\mathrm{Ca}^{2+}$ - and $\mathrm{Mg}^{2+}$-free phosphate-buffered saline (PBS). After the atria and aorta were excised, the ventricles were minced and enzymatically digested with $0.125 \%$ trypsin (Gibco BRL Life Technologies, Inc.) and $0.1 \%$ collagenase I (Sigma-Aldrich). The liberated cells were collected by centrifugation at $200 \mathrm{x} \mathrm{g}$ for $5 \mathrm{~min}$ at room temperature and incubated in a $100-\mathrm{mm}$ culture dish for $90 \mathrm{~min}$ at $37^{\circ} \mathrm{C}$ in an incubator with $5 \% \mathrm{CO}_{2}$ air. Non-adherent cells were harvested as cardiomyocytes. They were seeded at a density of $1 \times 10^{6}$ cells $/ \mathrm{ml}$ and cultured in DMEM supplemented with $10 \% \mathrm{FBS}$ and $0.1 \%$ penicillin/streptomycin at $37^{\circ} \mathrm{C}$ in an incubator with $5 \% \mathrm{CO}_{2}$. The medium was changed twice daily to maintain lactate at low concentrations in all groups for 2 days prior to experimentation. There were 4 experimental groups: i) the control group, ii) the AA group, iii) the lactate group and iv) the lactate + AA group. The cells were incubated with AA $(20 \mu \mathrm{M})$ for $24 \mathrm{~h}$ prior to stimulation with lactate $(20 \mathrm{mM})$ for $24 \mathrm{~h}$. Untreated cells served as the controls. AA was freshly prepared as a stock solution in DMSO and diluted with sterile double-distilled water [0.1\% (v/v) DMSO]. DMSO was present at equal concentrations $(0.03 \%)$ in all groups. Sodium lactate was dissolved in sterile double-distilled water.

Cell viability assay. Cardiomyocyte viability was measured by CCK-8 assay according to the manufacturer's instructions. In brief, cardiomyocytes were initially cultured at a density of $1 \times 10^{4}$ cells/well in 96-well plates. The cells were then incubated with various concentrations of AA (5-30 $\mu \mathrm{M})$ for $24 \mathrm{~h}$ or exposed to various concentrations of lactate (10-40 mM) for $24 \mathrm{~h}$ at $37^{\circ} \mathrm{C}$. CCK-8 solution $(10 \mu \mathrm{l})$ was subsequently added to each well and the culture was incubated for $4 \mathrm{~h}$ at $37^{\circ} \mathrm{C}$. The absorbance at $450 \mathrm{~nm}$ was measured using a microplate reader (Bio-Rad Laboratories, Hercules, CA, USA). All measurements were performed in triplicate, and cell viability was calculated as a percentage.

Determination of intracellular ROS levels. The levels of intracellular ROS were measured by flow cytometry using DCFH-DA as the probe. Cardiomyocytes were incubated with lactate for $24 \mathrm{~h}$ after being pre-treated with or without AA for $24 \mathrm{~h}$. The cells were then harvested and incubated with DCFH-DA $(10 \mu \mathrm{mol} / \mathrm{l})$ diluted in serum-free DMEM at $37^{\circ} \mathrm{C}$ for $30 \mathrm{~min}$. Subsequently, the cells were washed with PBS 3 times and analyzed by flow cytometry at an excitation wavelength of $488 \mathrm{~nm}$ and an emission wavelength of $530 \mathrm{~nm}$. Data analysis was performed using a Kaluza flow cytometer (Beckman Coulter, Brea, CA, USA).

Mitochondrial membrane potential $(\Delta \psi m)$ assay. $\Delta \psi \mathrm{m}$ was determined by flow cytometry and visualized by fluorescence microscopy using the fluorescent probe, JC-1. JC-1 accumulates in the mitochondria with a normal $\Delta \psi \mathrm{m}$, leading to the formation of JC-1 aggregates and the emission of red fluorescence. However, JC-1 leaks from the mitochondria into the cytoplasm as a monomer when the $\Delta \psi \mathrm{m}$ is depolarized, resulting in a decrease in red fluorescence and an increase in green fluorescence. Following treatment, the cardiomyocytes were washed with PBS and incubated in fresh medium containing $\mathrm{JC}-1(10 \mu \mathrm{g} / \mathrm{ml})$ for $20 \mathrm{~min}$ at $37^{\circ} \mathrm{C}$. The stained cells were visualized under a fluorescence microscope (Nikon, Tokyo, Japan). In addition, the cells in each group were also collected and resuspended in fresh medium containing JC-1 $(10 \mu \mathrm{g} / \mathrm{ml})$ and then incubated for $20 \mathrm{~min}$ at $37^{\circ} \mathrm{C}$. The ratio of red and green fluorescence was analyzed using a Kaluza flow cytometer.

Annexin V-FITC/PI apoptosis assay. Cell apoptosis was evaluated using an Annexin V-FITC/PI staining kit. In brief, the cardiomyocytes in each group were harvested and washed 3 times with PBS, and then resuspended in binding buffer. A total of $1 \times 10^{6}$ cells $/ \mathrm{ml}$ was transferred to a flow tube and then stained with $2 \mu \mathrm{l}$ of Annexin V-FITC and $2 \mu \mathrm{l}$ of PI for $15 \mathrm{~min}$ at room temperature in the dark. The samples were analyzed using a Kaluza flow cytometer. Annexin $\mathrm{V}^{+} / \mathrm{PI}^{-}$and 

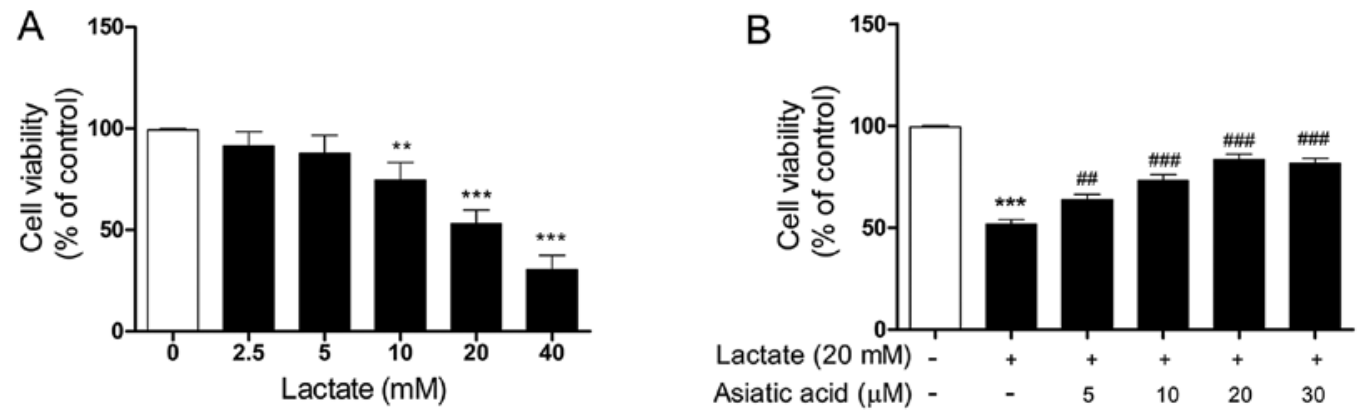

Figure 1. Protective effect of asiatic acid (AA) on lactate-induced cytotoxicity. (A) Cardiomyocytes were treated with various concentrations of lactate alone for $24 \mathrm{~h}$, and cell viability was determined by cell counting kit-8 (CCK-8) assay. (D) Cardiomyocytes were treated with various concentrations of AA (5-30 $\mu \mathrm{M})$ for $24 \mathrm{~h}$, and then exposed to $20 \mu \mathrm{M}$ lactate for $24 \mathrm{~h}$, and the cell viability was detected by CCK- 8 assay. Data are presented as the means \pm SD of 3 independent experiments. ${ }^{* *} \mathrm{P}<0.01$ and ${ }^{* * * *} \mathrm{P}<0.001$ vs. untreated cells; ${ }^{\# \#} \mathrm{P}<0.01$ and ${ }^{\# \# \#} \mathrm{P}<0.001$ vs. lactate-treated cells.

Annexin $\mathrm{V}^{+} / \mathrm{PI}^{+}$cells were considered as apoptotic cells in the early and late phase, respectively.

Terminal deoxynucleotidyltransferase-mediated dUTP nickend labeling (TUNEL) staining. For the detection of apoptosis, the cardiomyocytes were stained using the TUNEL technique with an in situ cell death detection kit according to the manufacturer's instructions. Briefly, the cultured cell smears were fixed with $4 \%$ paraformaldehyde in PBS for $1 \mathrm{~h}$ at room temperature and then permeabilized with $0.1 \%$ Triton $\mathrm{X}-100$ in $0.1 \%$ sodium citrate for $2 \mathrm{~min}$ on ice. The cells were then incubated with the TUNEL reaction mixture for $1 \mathrm{~h}$ at $37^{\circ} \mathrm{C}$. After rinsing with PBS, cells were stained with 4',6-diamidino-2-phenylindole (DAPI) for $5 \mathrm{~min}$ and visualized under a fluorescence microscope. The number of TUNEL-positive nuclei (green fluorescence) was expressed as a percentage of total nuclei (blue fluorescence).

Western blot analysis. Cytoplasmic and mitochondrial proteins were isolated from the cardiomyocytes by differential centrifugation using the mitochondrial fractionation kit according to the manufacturer's instructions. Briefly, the harvested cardiomyocytes were resuspended in ice-cold cytoplasmic lysis buffer for 10 min and homogenized on ice by an ultrasonic homogenizer. The homogenate was centrifuged at $800 \mathrm{x}$ g for $10 \mathrm{~min}$ at $4^{\circ} \mathrm{C}$. The collected supernatant was centrifuged at $10,000 \mathrm{x} \mathrm{g}$ for $20 \mathrm{~min}$ at $4^{\circ} \mathrm{C}$ to obtain the cytoplasmic protein (supernatant) and the mitochondrial fraction (pellet). The mitochondrial fraction was then resuspended in the mitochondria lysis buffer on ice for $20 \mathrm{~min}$. The supernatant (mitochondrial protein) was collected by centrifugation at $10,000 \mathrm{x}$ g for $10 \mathrm{~min}$ at $4^{\circ} \mathrm{C}$. The protein concentrations were determined by the bicinchoninic acid (BCA) method. The proteins (20-30 $\mu \mathrm{g})$ were separated by $10-15 \%$ sodium dodecyl sulfate-polyacrylamide gel electrophoresis (SDS-PAGE) and transferred onto polyvinylidine difluoride membranes. After blocking in $5 \%(\mathrm{v} / \mathrm{v})$ non-fat dry milk in Tris-buffered saline (TBS) with $0.1 \%$ Tween-20 (TBST) at room temperature for $2 \mathrm{~h}$, the membranes were then incubated with primary antibodies overnight at $4^{\circ} \mathrm{C}$. After washing with TBST, the membranes were incubated with HRP-conjugated secondary antibodies at room temperature for $1 \mathrm{~h}$. Blots were developed using ECL reagents (Thermo Scientific, Rockford, IL, USA) and were then exposed using the digital imaging system (Bio-Rad Laboratories). The intensity of each band was analyzed using Image Lab 2.0 software (Bio-Rad Laboratories). The intensity of mitochondrial MCT1 was normalized to that of COX4. The intensities of cytoplasmic cleaved caspase-9, cleaved caspase-3 and cytochrome $c$ were normalized to those of $\beta$-actin.

Statistical analysis. All results are presented as the mean \pm standard deviation (SD). The GraphPad Prism 5.01 software (GraphPad Software, Inc., CA, USA) and the PASW Statistics 18.0 (SPSS Inc., Fayetteville, NC, USA) packages were used. Differences among groups were tested by one-way ANOVA. Comparisons between 2 groups were performed by an unpaired two-tailed Student's t-test. A value of $\mathrm{P}<0.05$ was considered to indicate a statistically significant difference.

\section{Results}

AA protects cardiomyocytes against lactate-induced damage. The effect of lactate on cell viability was first evaluated. As shown in Fig. 1A, cells were treated with increasing concentrations of lactate for $24 \mathrm{~h}$. Lactate impaired cell viability in a concentration-dependent manner over the tested concentration range (10-40 mM). We used $20 \mathrm{mM}$ lactate for $24 \mathrm{~h}$ in our subsequent experiments, as this concentration of lactate caused significant cell damage and reduced cell viability by approximately $50 \%$ relative to the control cells. We then evaluated the effects of AA on lactate-induced cell damage. The cardiomyocytes were treated with lactate $(20 \mathrm{mM})$ for $24 \mathrm{~h}$ in the presence or absence of AA (5-30 $\mu \mathrm{M})$. No noticeable changes were observed in the viability of the cardiomyocytes treated with AA (5-30 $\mu \mathrm{M})$ for $24 \mathrm{~h}$, as previously described by $\mathrm{Si}$ et al (23). The cytotoxic effects of lactate on cardiomyocytes were significantly attenuated by pre-treatment with AA $(5-30 \mu \mathrm{M})$ for $24 \mathrm{~h}$, and AA at $20 \mu \mathrm{M}$ provided maximal protection against lactate-induced damage (Fig. 1B).

AA inhibits the lactate-induced increase in intracellular ROS levels. The intracellular ROS levels were measured by flow cytometry using DCFH-DA as the probe. As shown in Fig. 2, when the cardiomyocytes were exposed to $20 \mathrm{mM}$ lactate for $24 \mathrm{~h}$, the intracellular ROS levels were substantially increased compared with those of the untreated cells, revealing that lactate enhanced intracellular ROS generation. Pre-treatment with AA attenuated the excess generation of ROS caused by lactate. However, AA $(20 \mu \mathrm{M})$ alone did not affect the intracellular ROS levels. 

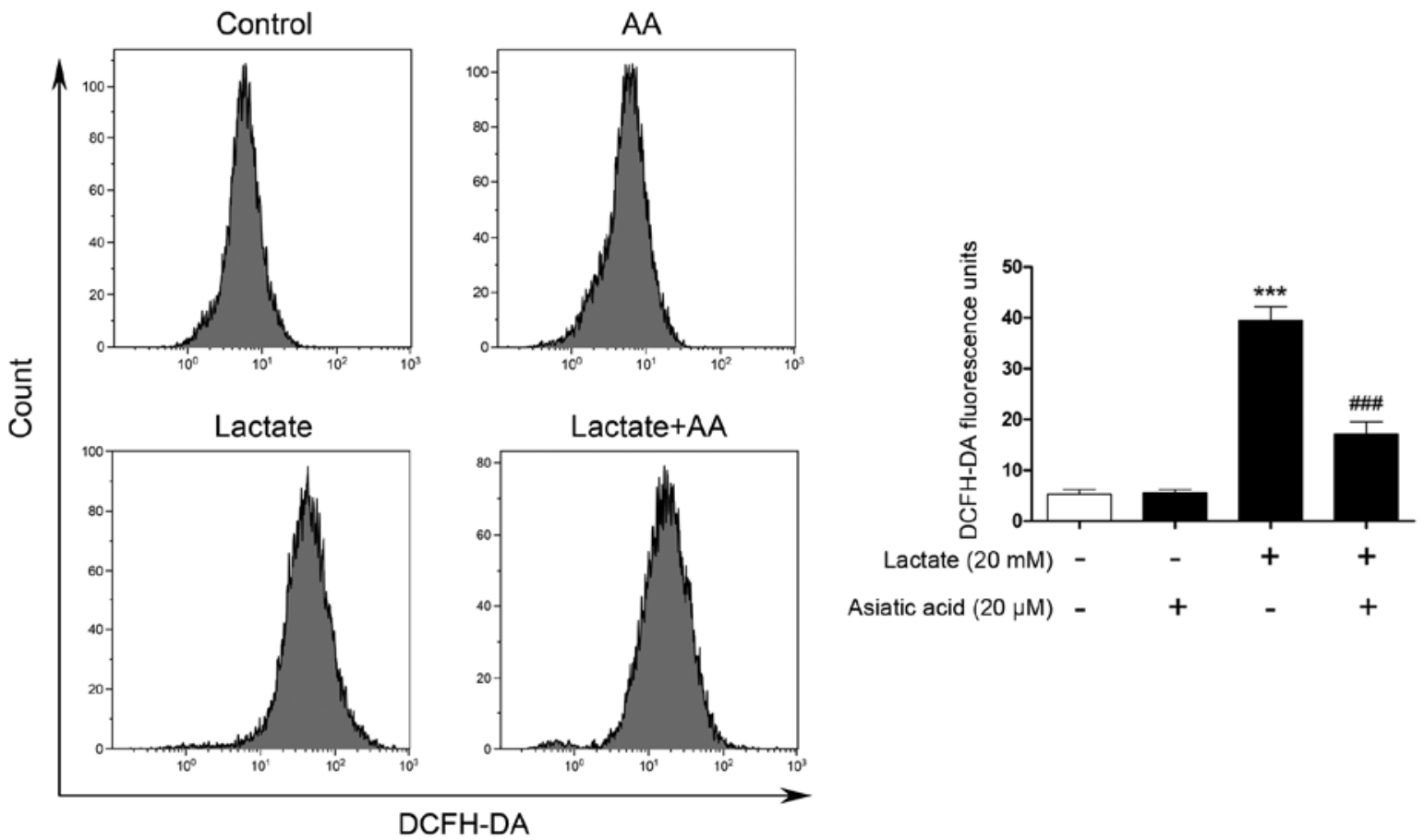

Figure 2. Asiatic acid (AA) reduces the lactate-induced increase in intracellular reactive oxygen species (ROS) levels. Cells were treated with AA (20 $\mu \mathrm{M})$ for $24 \mathrm{~h}$ and then exposed to lactate $(20 \mathrm{mM})$ for $24 \mathrm{~h}$. Intracellular ROS levels were measured by flow cytometry using 2',7'-dichlorofluorescein-diacetate (DCFH-DA). Data are presented as the means \pm SD of 3 independent experiments. ${ }^{* * *} \mathrm{P}<0.001$ vs. untreated cells; ${ }^{\# \#} \mathrm{P}<0.001$ vs. lactate-treated cells.

AA prevents the lactate-induced reduction in $\Delta \psi m . \Delta \psi \mathrm{m}$ was examined by JC-1 staining and the loss of $\Delta \psi \mathrm{m}$ was analyzed by both fluorescence microscopy and flow cytometry. Stimulation with lactate resulted in a decrease in red fluorescence and an increase in green fluorescence in the cardiomyocytes, compared with the control cells (Fig. 3A). Pre-treatment with AA reversed the dissipation of $\Delta \psi \mathrm{m}$ induced by lactate. Similarly, as shown by flow cotymetry, the loss of $\Delta \psi \mathrm{m}$ was substantially increased when the cardiomyocytes were exposed to $20 \mathrm{mM}$ lactate for $24 \mathrm{~h}$ and the lactate-induced loss of $\Delta \psi \mathrm{m}$ was counteracted by pre-treatment with AA (Fig. 3B).

AA protects cardiomyocytes against lactate-induced apoptosis. TUNEL-FITC staining was used to evaluate morphological changes due to the apoptosis of cardiomyocytes. Following exposure to $20 \mathrm{mM}$ lactate for $24 \mathrm{~h}$, the percentage of apoptotic nuclei was markedly increased compared with the untreated nuclei. By contrast, pre-treatment with AA significantly reduced the number of TUNEL-positive nuclei induced by lactate (Fig. 4). Apoptosis was also confirmed by flow cytometry. Following exposure to $20 \mathrm{mM}$ lactate for $24 \mathrm{~h}$, the percentage of apoptotic cells was significantly increased compared with the untreated cells. By contrast, pre-treatment with AA substantially reduced the rate of apoptosis by $54.51 \%$ compared with lactate stimulation alone (Fig. 5).

AA regulates the lactate-induced signaling cascade. Following exposure to $20 \mathrm{mM}$ lactate for $24 \mathrm{~h}$, the expression of mitochondrial MCT1 was significantly increased compared with the untreated cells. Moreover, pre-treatment with AA further increased the expression of mitochondrial MCT1 induced by lactate (Fig. 6A). However, treatment with AA (20 $\mu \mathrm{M})$ alone had no effect on the expression of mitochondrial MCT1.
With the collapse of $\Delta \psi \mathrm{m}$, cytochrome $c$ can activate the caspase responsible for apoptosis when it is released into the cytosol from the mitochondria. The expression levels of cytoplasmic cytochrome $c$, cleaved caspase- 9 and caspase- 3 were markedly increased following stimulation with lactate (Fig. 6B). By contrast, pre-treatment with AA substantially reduced the lactate-induced increase in the expression of cytoplasmic cytochrome $c$, cleaved caspase- 9 and caspase-3.

\section{Discussion}

In the present study, we examined the effect of AA on lactateinduced cardiomyocyte apoptosis in vitro. We found that AA protected the cardiomyocytes against lactate-induced apoptosis by regulating the lactate signaling cascade, involving the inhibition of oxidative stress and mitochondria-dependent caspase activation, as well as the upregulation of mitochondrial MCT1 expression. Our results indicated that AA is a suitable candidate for the prevention and treatment of cardiomyocyte apoptosis.

In conventional wisdom, lactate is formed as the result of oxygen insufficiency and utilized under fully aerobic conditions in myocardial mitochondria. Lactate can be transported across lipid bilayer membranes by monocarboxylate transport (MCT) proteins (10). MCT1 is expressed abundantly in rodent and human hearts, and is localized in sarcolemmal and mitochondrial membranes $(10,25,26)$. Moreover, the co-localization of MCT1, lactate dehydrogenase (LDH), CD147 and COX in the mitochondrial membrane has been demonstrated by Hashimoto et al (27) and is described as the mitochondrial lactate oxidation complex. As part of the intracellular lactate shuttle mechanism, the presence of MCT1 allows mitochondrial lactate influx and oxidation. However, during exercise or pathological conditions, such as hypoxia and ischemia, heightened glycolysis causes 

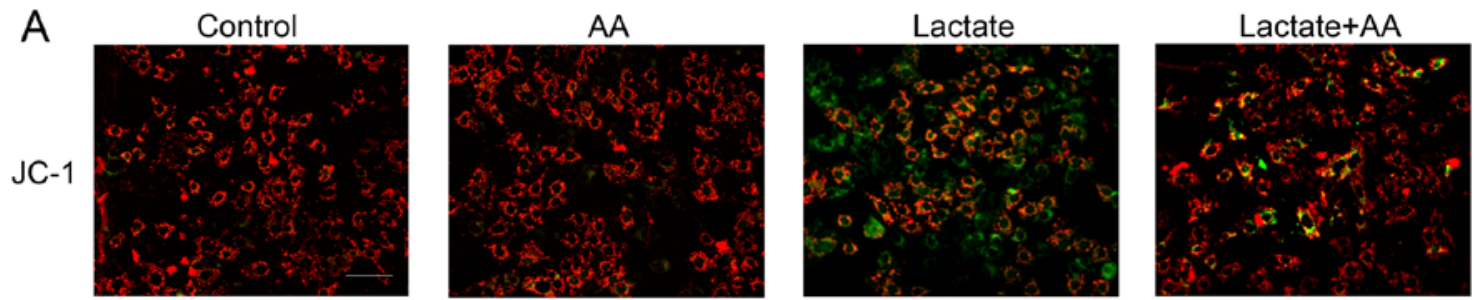

B
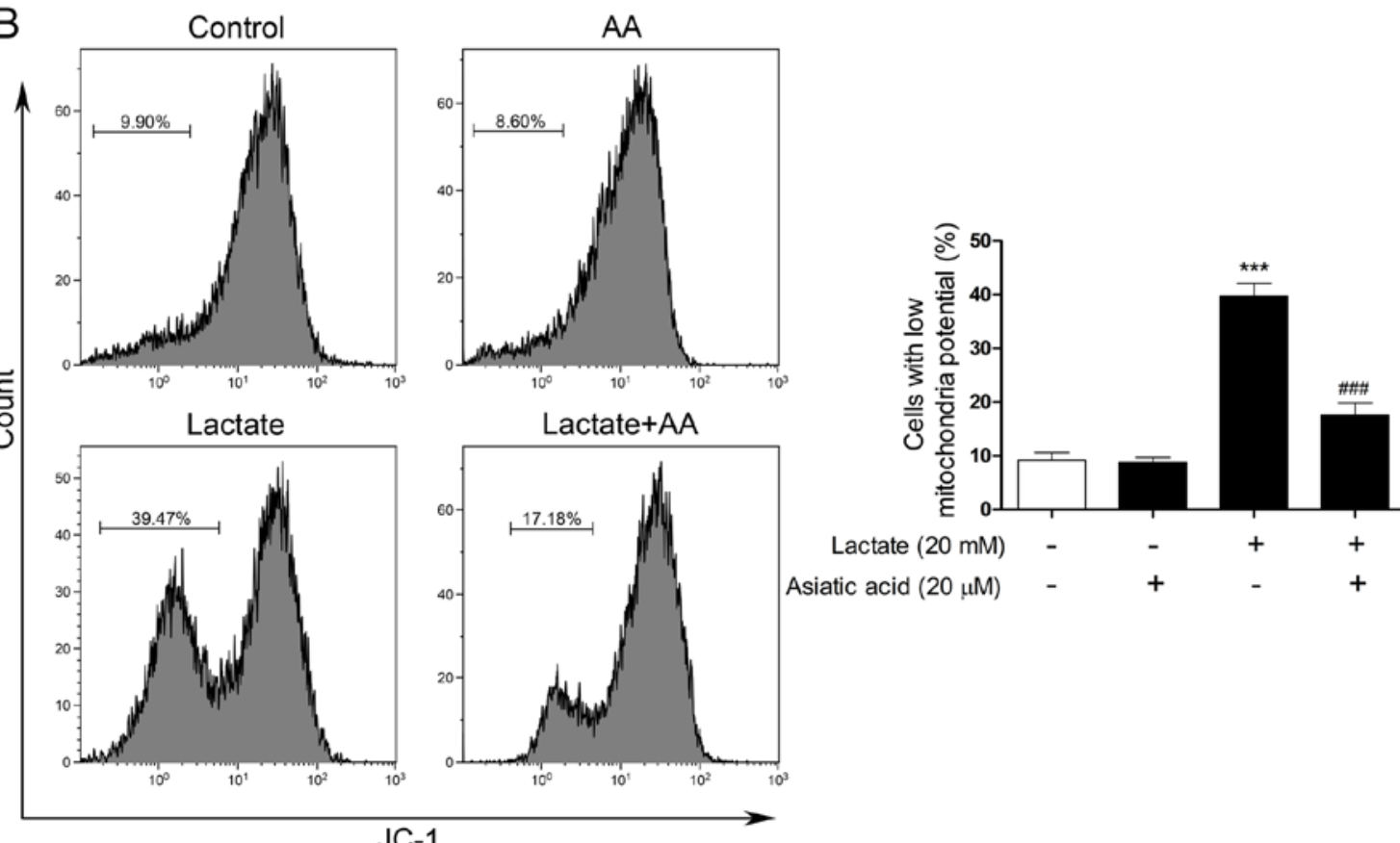

Figure 3. Asiatic acid (AA) prevents lactate-induced reduction in the mitochondrial membrane potential ( $\Delta \psi \mathrm{m})$. (A) The $\Delta \psi \mathrm{m}$ was visualized by fluorescence microscopy using the JC-1 probe. Red fluorescence represents JC-1 aggregates formed in normal cells with high $\Delta \psi \mathrm{m}$, whereas green fluorescence represents JC-1 monomers in cells with a low $\Delta \psi \mathrm{m}$. Scale bar, $100 \mu \mathrm{m}$. (B) The $\Delta \psi \mathrm{m}$ was measured by flow cytometry using the JC-1 probe. Data are presented as the means \pm SD of 3 independent experiments. ${ }^{* * *} \mathrm{P}<0.001$ vs. untreated cells; ${ }^{\# \# \#} \mathrm{P}<0.001$ vs. lactate-treated cells.
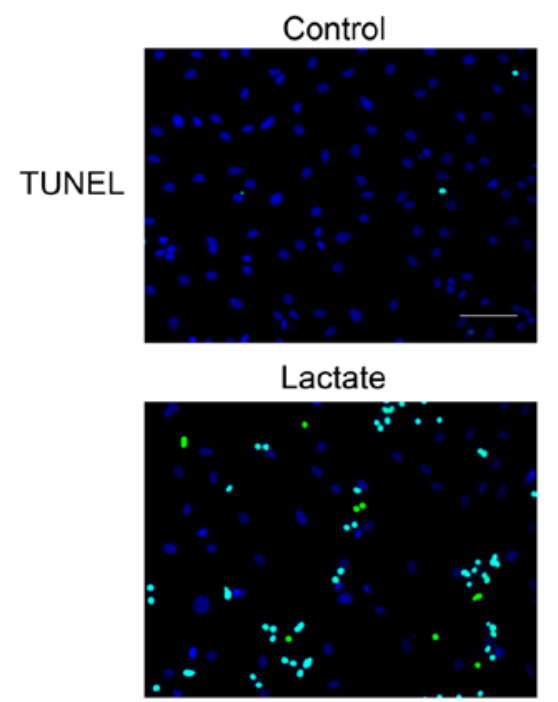
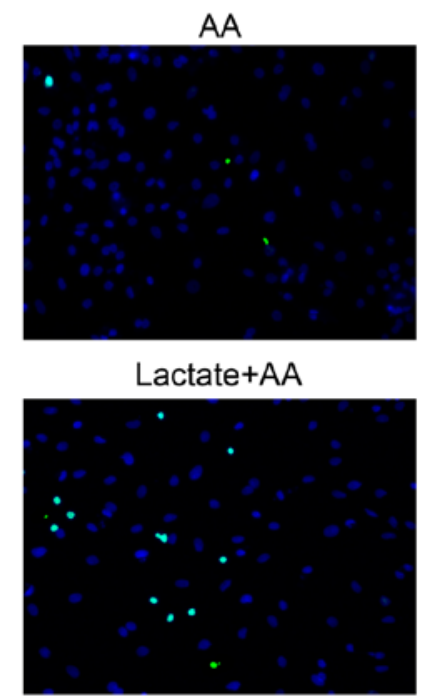

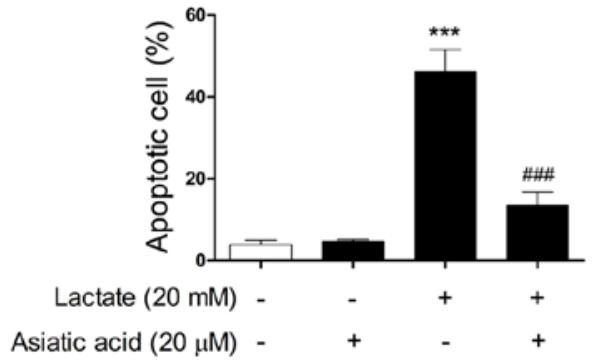

Asiatic acid $(20 \mu \mathrm{M})$ -

Figure 4. Asiatic acid (AA) protects cardiomyocytes against lactate-induced apoptosis. Cardiomyocyte apoptosis was examined by TUNEL-FITC staining and visualized by fluorescence microscopy. The number of TUNEL-positive nuclei (green fluorescence) was expressed as a percentage of total nuclei (blue fluorescence). Data are presented as the means \pm SD of 3 independent experiments. Scale bar, $100 \mu \mathrm{m}$. ${ }^{* * *} \mathrm{P}<0.001$ vs. untreated cells; ${ }^{\# \# \# P<0.001 ~ v s . ~ l a c t a t e-t r e a t e d ~ c e l l s ~}$

a surge in lactate production. The corresponding increased expression of MCT1 helps to attenuate intracellular acidification. Previous studies have identified a convincingly positive correlation between the dysfunction of lactate metabolism and cardiovascular disorders. A maximal lactate influx into cardiomyocytes and increased MCT1 expression has been observed 


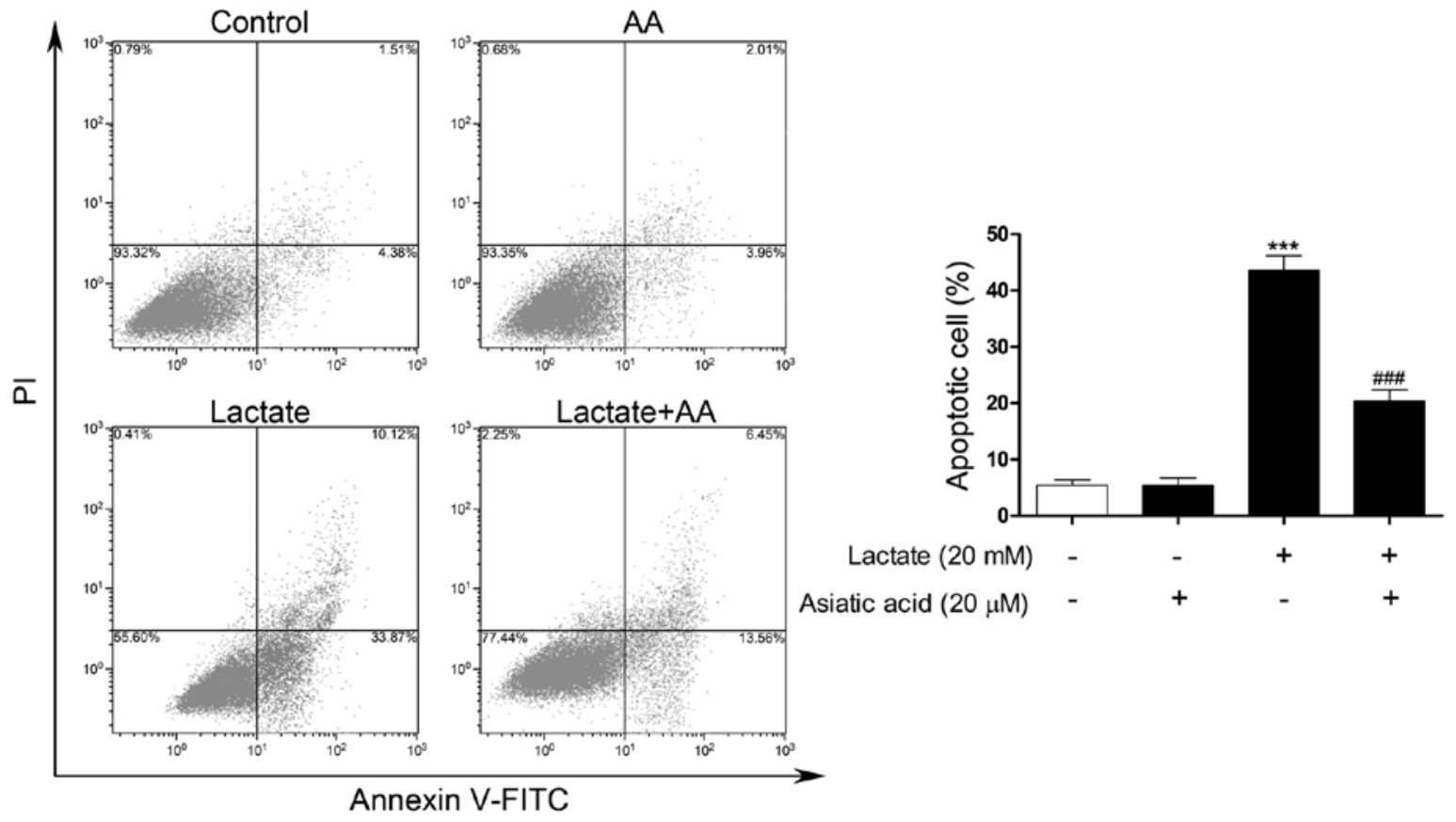

Figure 5. Asiatic acid (AA) inhibits lactate-induced cardiomyocytes apoptosis. Cardiomyocyte apoptosis was examined by Annexin V-FITC/propidium iodide (PI) staining and analyzed by flow cytometry. Data are presented as the means \pm SD of 3 independent experiments. ${ }^{* * * *} \mathrm{P}<0.001$ vs. untreated cells;

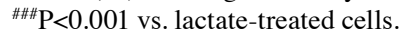

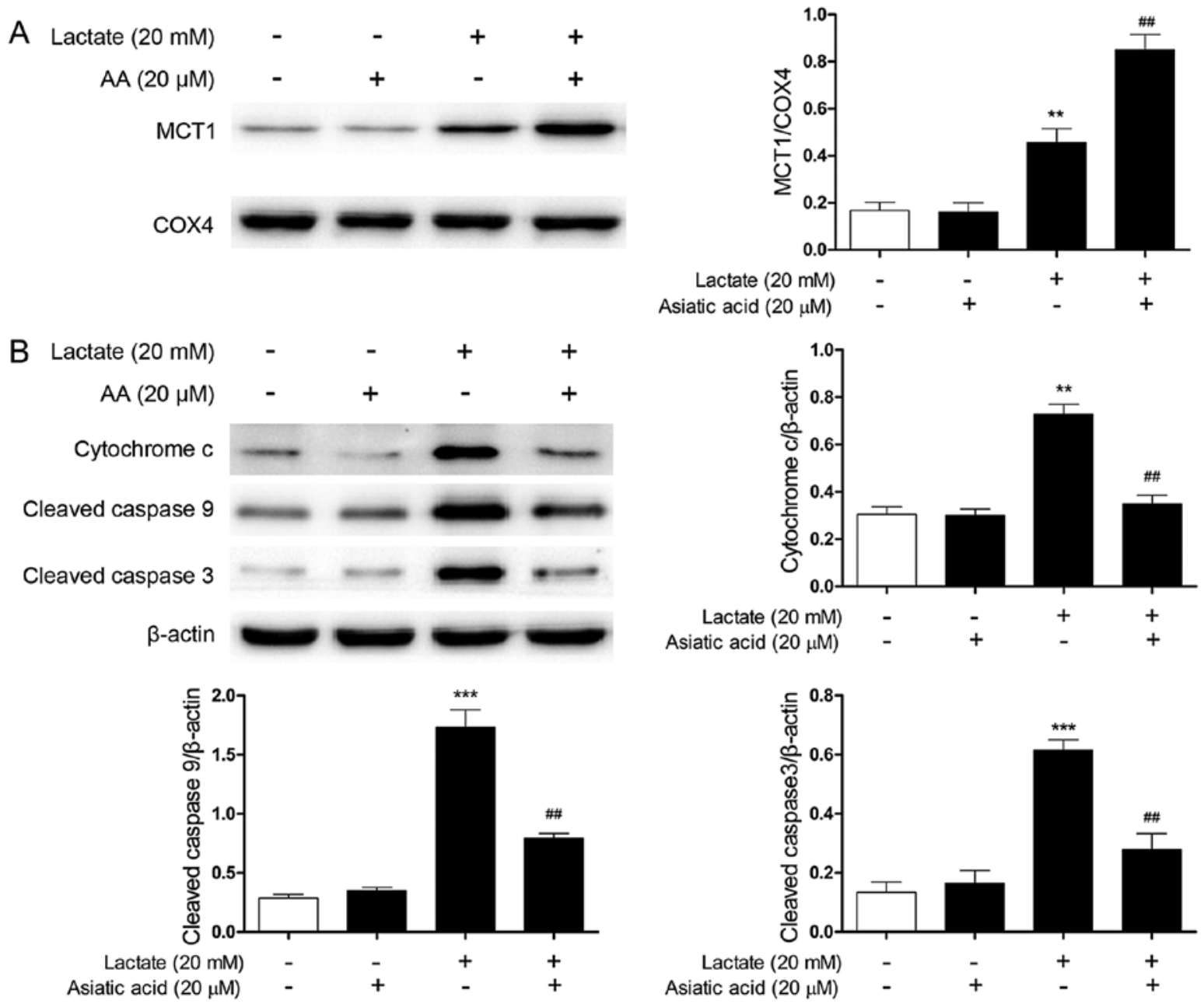

Figure 6. Asiatic acid (AA) regulates lactate-induced signaling cascade. (A) The expression of mitochondrial monocarboxylate transporter 1 (MCT1) was assayed by western blot analysis. (B) The expression of cytoplasmic cytochrome $c$, cleaved caspase- 9 and caspase-3 were assayed by western blot analysis. Data are presented as the means \pm SD of 3 independent experiments. ${ }^{* *} \mathrm{P}<0.01$ and ${ }^{* * * *} \mathrm{P}<0.001$ vs. untreated cells; ${ }^{\# \#} \mathrm{P}<0.01$ vs. lactate-treated cells. 
both in rat models of myocardial infarction (15) and volume overload (18). In patients with atrial fibrillation, the upregulation of cytoplasmic lactate concentration and mitochondrial MCT1 expression has also been confirmed in right atrial appendage tissues (RAAs) (17). These findings reveal that the excessive accumulation of lactate and the increased expression of MCT1 concurrs in cardiovascular diseases. Conversely, the blockade of MCT1 function by its competitive inhibitor in a mouse model of ischemia/reperfusion injury has been shown to decrease cardiac performance and increase the infarct size. In vitro, the MCT1 inhibitor also increased the death of HL-1 cells stimulated with hydrogen peroxide (28). In the present study, we found that the expression of mitochondrial MCT1 was significantly increased in lactate-stimulated cardiomyocytes in vitro. Pre-treatment with AA further increased the expression of mitochondrial MCT1 induced by lactate and inhibited cardiomyocyte apoptosis. Taken together, these findings indicate that lactate may have an independent effect on the development of cardiovascular diseases.

Rather than an end-product of glycolysis during oxygen insufficiency, we now know that lactate is produced continuously under fully aerobic conditions (12). Previous studies have confirmed that lactate is an important cell-signaling molecule involved in the regulation of the cell redox state and related signaling cascade. For instance, a low level of exogenous lactate can rapidly activate ROS production, upregulate mitochondrial MCT1 expression and increase mitochondrial biogenesis in L6 cells by activating several transcription factors (29). However, in $\mathrm{CHO}$ cells, lactate-induced cytosolic acidification has been shown to lead to mitochondrial-dependent apoptosis, including the reduction of $\Delta \psi \mathrm{m}$, the release of cytochrome $c$, and an increase in caspase-3 enzymatic activity (30). Moreover, the ROS levels and mitochondrial-dependent apoptotic proteins were also increased consistent with the elevated lactate concentration in RAAs in atrial fibrillation (17). Conversely, Kubasiak et al (31) reported that hypoxia did not induce cardiomyocyte apoptosis in the absence of lactate acidosis. In the present study, we observed that stimulation with lactate significantly increased intracellular ROS levels and activated mitochondrial-dependent apoptosis, including the reduction of $\Delta \psi \mathrm{m}$, the release of cytochrome $c$, and the upregulation of cleaved caspase- 9 and caspase- 3 expression. Our data suggest that lactate signaling plays a key role in mitochondrial energy metabolism and the apoptosis of cardiomyocytes. Although the increased expression of mitochondrial MCT1 facilitates lactate oxidation from the cytosol to the mitochondria, lactic acidosis still occurs inevitably, thereby resulting in oxidative stress and mitochondrial-dependent apoptosis. However, pre-treatment with AA substantially attenuated oxidative stress, preserved the $\Delta \psi \mathrm{m}$, and inhibited cardiomyocyte apoptosis induced by lactate.

In conclusion, to the very best of our knowledge, the present study is the first to demonstrate that AA inhibits the lactate-induced cardiomyocyte apoptosis. The mechanisms through which AA inhibits this apoptotic process involve the regulation of the lactate signaling cascade, including the inhibition of oxidative stress and mitochondrial-dependent caspase activation, as well as the upregulation of mitochondrial MCT1 expression, thereby relieving lactate acidosis and inhibiting cardiomyocyte apoptosis. Our results suggest that AA may be used as a therapeutic agent for the prevention and treatment of cardiomyocyte apoptosis. Further studies are warranted to determine its biological efficacy and precise mechanisms of action in vitro and in vivo.

\section{Acknowledgements}

This study was funded by the Jiangsu Province's Outstanding Medical Academic Leader Program (grant no. LJ201140).

\section{References}

1. Saraste A, Pulkki K, Kallajoki M, Henriksen K, Parvinen M and Voipio-Pulkki LM: Apoptosis in human acute myocardial infarction. Circulation 95: 320-323, 1997.

2. Gao HK, Yin Z, Zhou N, Feng XY, Gao F and Wang HC: Glycogen synthase kinase 3 inhibition protects the heart from acute ischemia-reperfusion injury via inhibition of inflammation and apoptosis. J Cardiovasc Pharmacol 52: 286-292, 2008.

3. Aharinejad S, Andrukhova O, Lucas T, Zuckermann A, Wieselthaler G, Wolner E and Grimm M: Programmed cell death in idiopathic dilated cardiomyopathy is mediated by suppression of the apoptosis inhibitor Apollon. Ann Thorac Surg 86: 109-114, discussion 114, 2008.

4. Narula J, Haider N, Virmani R, DiSalvo TG, Kolodgie FD, Hajjar RJ, Schmidt U, Semigran MJ, Dec GW and Khaw BA: Apoptosis in myocytes in end-stage heart failure. N Engl $\mathrm{J}$ Med 335: 1182-1189, 1996.

5. Bryant D, Becker L, Richardson J, Shelton J, Franco F, Peshock R, Thompson $\mathrm{M}$ and Giroir B: Cardiac failure in transgenic mice with myocardial expression of tumor necrosis factor-alpha. Circulation 97: 1375-1381, 1998.

6. Sayen MR, Gustafsson AB, Sussman MA, Molkentin JD and Gottlieb RA: Calcineurin transgenic mice have mitochondrial dysfunction and elevated superoxide production. Am J Physiol Cell Physiol 284: C562-C570, 2003.

7. Wang J, Silva JP, Gustafsson CM, Rustin P and Larsson NG: Increased in vivo apoptosis in cells lacking mitochondrial DNA gene expression. Proc Natl Acad Sci USA 98: 4038-4043, 2001.

8. Crow MT, Mani K, Nam YJ and Kitsis RN: The mitochondrial death pathway and cardiac myocyte apoptosis. Circ Res 95: 957-970, 2004

9. Lee Y and Gustafsson AB: Role of apoptosis in cardiovascular disease. Apoptosis 14: 536-548, 2009.

10. Halestrap AP and Wilson MC: The monocarboxylate transporter family - role and regulation. IUBMB Life 64: 109-119, 2012.

11. Brooks GA, Dubouchaud H, Brown M, Sicurello JP and Butz CE: Role of mitochondrial lactate dehydrogenase and lactate oxidation in the intracellular lactate shuttle. Proc Natl Acad Sci USA 96: 1129-1134, 1999.

12. Gladden LB: Lactate metabolism: A new paradigm for the third millennium. J Physiol 558: 5-30, 2004.

13. Bonen A: The expression of lactate transporters (MCT1 and MCT4) in heart and muscle. Eur J Appl Physiol 86: 6-11, 2001.

14. Hashimoto T and Brooks GA: Mitochondrial lactate oxidation complex and an adaptive role for lactate production. Med Sci Sports Exerc 40: 486-494, 2008.

15. Jóhannsson E, Lunde PK, Heddle C, Sjaastad I, Thomas MJ, Bergersen L, Halestrap AP, Blackstad TW, Ottersen OP and Sejersted OM: Upregulation of the cardiac monocarboxylate transporter MCT1 in a rat model of congestive heart failure. Circulation 104: 729-734, 2001.

16. Halestrap AP, Wang X, Poole RC, Jackson VN and Price NT: Lactate transport in heart in relation to myocardial ischemia. Am J Cardiol 80: 17A-25A, 1997.

17. Xu J, Xu X, Si L, Xue L, Zhang S, Qin J, Wu Y, Shao Y, Chen Y and Wang $X$ : Intracellular lactate signaling cascade in atrial remodeling of mitral valvular patients with atrial fibrillation. J Cardiothorac Surg 8: 34, 2013.

18. Evans RK, Schwartz DD and Gladden LB: Effect of myocardial volume overload and heart failure on lactate transport into isolated cardiac myocytes. J Appl Physiol (1985) 94: 1169-1176, 2003.

19. Pittella F, Dutra RC, Junior DD, Lopes MT and Barbosa NR: Antioxidant and cytotoxic activities of Centella asiatica (L) Urb. Int J Mol Sci 10: 3713-3721, 2009. 
20. Yun KJ, Kim JY, Kim JB, Lee KW, Jeong SY, Park HJ, Jung HJ, Cho YW, Yun K and Lee KT: Inhibition of LPS-induced NO and PGE2 production by asiatic acid via NF-kappa B inactivation in RAW 264.7 macrophages: Possible involvement of the IKK and MAPK pathways. Int Immunopharmacol 8: 431-441, 2008.

21. Krishnamurthy RG, Senut MC, Zemke D, Min J, Frenkel MB Greenberg EJ, Yu SW, Ahn N, Goudreau J, Kassab M, et al: Asiatic acid, a pentacyclic triterpene from Centella asiatica, is neuroprotective in a mouse model of focal cerebral ischemia. J Neurosci Res 87: 2541-2550, 2009.

22. Tang LX, He RH, Yang G, Tan JJ, Zhou L, Meng XM, Huang XR and Lan HY: Asiatic acid inhibits liver fibrosis by blocking TGF-beta/Smad signaling in vivo and in vitro. PLoS One 7: e31350, 2012.

23. Si L, Xu J, Yi C, Xu X, Wang F, Gu W, Zhang Y and Wang X: Asiatic acid attenuates cardiac hypertrophy by blocking transforming growth factor- $\beta 1$-mediated hypertrophic signaling in vitro and in vivo. Int J Mol Med 34: 499-506, 2014.

24. Zhang X, Wu J, Dou Y, Xia B, Rong W, Rimbach G and Lou Y: Asiatic acid protects primary neurons against $\mathrm{C} 2$-ceramideinduced apoptosis. Eur J Pharmacol 679: 51-59, 2012.

25. Brooks GA, Brown MA, Butz CE, Sicurello JP and Dubouchaud H: Cardiac and skeletal muscle mitochondria have a monocarboxylate transporter MCT1. J Appl Physiol (1985) 87: 1713-1718, 1999.
26. Butz CE, McClelland GB and Brooks GA: MCT1 confirmed in rat striated muscle mitochondria. J Appl Physiol (1985) 97: 1059-1066, 2004

27. Hashimoto T, Hussien R and Brooks GA: Colocalization of MCT1, CD147, and LDH in mitochondrial inner membrane of L6 muscle cells: Evidence of a mitochondrial lactate oxidation complex. Am J Physiol Endocrinol Metab 290: E1237-E1244, 2006.

28. Martinov V, Rizvi SM, Weiseth SA, Sagave J, Bergersen LH and Valen G: Increased expression of monocarboxylate transporter 1 after acute ischemia of isolated, perfused mouse hearts. Life Sci 85: 379-385, 2009.

29. Hashimoto T, Hussien R, Oommen S, Gohil K and Brooks GA: Lactate sensitive transcription factor network in L6 cells: Activation of MCT1 and mitochondrial biogenesis. FASEB J 21: 2602-2612, 2007.

30. Jeong D, Kim TS, Lee JW, Kim KT, Kim HJ, Kim IH and Kim IY: Blocking of acidosis-mediated apoptosis by a reduction of lactate dehydrogenase activity through antisense mRNA expression. Biochem Biophys Res Commun 289: 1141-1149, 2001.

31. Kubasiak LA, Hernandez OM, Bishopric NH and Webster KA: Hypoxia and acidosis activate cardiac myocyte death through the Bcl-2 family protein BNIP3. Proc Natl Acad Sci USA 99: 12825-12830, 2002. 